\title{
Absolute Hidden Symmetry in Time and Absolute Asymmetry in Mass and Velocity between a Particle and Its Anti-Particle
}

\author{
Eyal Brodet ${ }^{1}$ \\ ${ }^{1}$ Jerusalem, 93707, Israel \\ Correspondence: Eyal Brodet, Jerusalem, 93707, Israel. E-mail: eyalbrodet@hotmail.com \\ Received: February 24, 2019 \\ Accepted: March 8, 2019 \\ Online Published: March 12, 2019 \\ doi:10.5539/apr.v11n2p43 \\ URL: http://dx.doi.org/10.5539/apr.v11n2p43
}

\begin{abstract}
In this paper we will the discuss possible hidden symmetry in time and the possible asymmetry in mass and velocity between a particle and its anti-particle. Possible hidden symmetry in time between a particle and its anti-particle manifested in their corresponding possible hidden variables in time, $f_{r}$ and $-f_{r}$ was first discussed in Brodet (2017b). In this paper we will extend the discussion and discuss how the above possible hidden symmetry in time and its corresponding possible symmetry in absolute position, energy and momentum, may yield an asymmetry in mass and velocity of a particle and its corresponding anti-particle. We will deconstruct the particle's and antiparticle's absolute position, into three complex number describing the particle/anti-particle time, velocity and mass. The particle/anti-particle symmetry and asymmetry of the above complex numbers will be discussed in the context of parity violation and the known asymmetry in angular production of particles and anti-particles. Moreover, the possible mass asymmetry will be used to explain the CP violation in the neutral kaon system. Finally, experimental ways to investigate and test the above are presented.
\end{abstract}

Keywords: Absolute Symmetry, Asymmetry, Time, Parity, CP, Violation

\section{Introduction}

In this paper we will discuss the possible hidden symmetry in time and the possible asymmetry in mass and velocity between a particle and its anti-particle. A central concept in the discussion of this paper is the possibility of a hidden variable in time, $f_{r}$, that was first introduced in Brodet (2010). As discussed in Brodet (2010), $f_{r(i)}$ was a value connected to each decay time value,$t_{i}$, that was suggested to govern particle's i decay time. i.e. the idea was to replace the random explanation of the decay time process by a deterministic explanation in which, a group of muon particles for example, are not identical but have a range of $f_{r}$ values that explain the observed muons decay time distribution. Following the above, possible hidden symmetry in time was first discussed in Brodet (2017b), where it was suggested that there may be a symmetry between the particle/anti-particle hidden variable in time, $f_{r}$ and $-f_{r}$. The idea was to first attempt to link deterministically between the particle's decay time distribution and its corresponding Breit-Wigner distribution by using the particle possible hidden variable in time $f_{r}$. Next, to suggest a symmetry between a particle and its anti-particle, by the division of the decay time 
distribution and its corresponding Breit-Wigner distribution into two parts, which correspond to positive $f_{r}$ and negative $-f_{r}$. This is where for each decay time $t_{i}$, there are $f_{r(i)}$ and $-f_{r(i)}$ that correspond to the same decay time, $t_{i}$, but to different values of masses, M1 and M2 in the Breit-Wigner distribution as shown in figure

1. Moreover, it was suggested, that $f_{r}$ among other variables, may be used to define the particle's absolute position, and be a part of a symmetry in a particle/anti-particle absolute position. Therefore, the suggested symmetry in time may be a part of a larger suggested symmetry between the particle/anti-particle absolute position (Brodet, 2018), which also yields a symmetry in energy and momentum. Note that the particle/anti-particle absolute position is defined as a point in the angular momentum dimension which is regarded here to be the most suitable dimension as it allows the inclusion of all the relevant variables. The discussion in this paper will be based on the above symmetries but will extend the discussion into the possible asymmetry that is yielded from the above symmetry requirements. More specifically, we will discuss how the above symmetry requirements may yield an asymmetry between particle/anti-particle in mass and velocity. We will show how one may deconstruct the absolute position, $P_{i(a b s)}$ of a particle/anti-particle into three complex numbers namely time, mean lifetime and velocity complex numbers. This is where there is a symmetry at rest in the time complex number and asymmetry in the mean lifetime and velocity complex numbers between a particle and its anti-particle. Moreover, using the time complex number, we may identify an alternative expression for the particle's hidden variable, $f_{r}$, which may provide a constraint on the particle/anti particle existence time as defined in Brodet (2010). Next, we will discuss how the asymmetry properties of the mean lifetime and velocity complex numbers, may help to explain the parity violation results as manifested in particle/anti-particle angular production as observed in $e^{+} e^{-} \rightarrow \mu^{+} \mu^{-}$ process at LEP. Moreover, the possible mass asymmetry (shown in the mean lifetime complex number) will be used to explain the $\mathrm{CP}$ violation in the neutral kaon system. Finally, experimental ways to test and investigate the above are presented.

This paper is divided into four sections. Section 2.1 describes the absolute position of a particle/anti-particle and its symmetric characteristics. Section 2.2 describes the possible deconstruction of $P_{i(a b s)}$, into three complex numbers, its symmetric and asymmetric nature and its possible implication on parity and CP violation. Section 2.3 discusses the particle's existence time. Section 3 discusses possible experimental ways to investigate and test the possible asymmetric nature of mass and velocity. Section 4 presents the conclusions.

\subsection{The Particle/Anti-Particle Absolute Position}

There may be two definitions for the particle/anti-particle absolute position as previously discussed in Brodet (2018). The first , $P_{i(r e s t) \_(a b s)}$, is the absolute position that doesn't depend on velocity:

$$
\left.P_{i(\text { rest_abs) }}=\frac{M_{i}}{t_{i(\text { mean })}}\left(c_{i(0)}^{2} t_{i(0)}^{2}\right)=\frac{M_{i}}{t_{i(\text { mean })}} \frac{\gamma_{i(a b s)}^{2}}{2}\left(V_{i(r))} t_{i(0)}\right)^{2}-\frac{\gamma_{i(a b s)}^{2}}{2}\left(c_{i} t_{i(0)}\right)^{2}\right)
$$

and a particle's absolute position that depends on its velocity: 


$$
P_{i(a b s)}=\frac{M_{i}}{t_{i(\text { mean })}} \gamma_{i(a b s)}^{2} V_{i\left(T\left(V_{r}\right)\right)}^{2} t_{i(0)}^{2}=\frac{M_{i}}{t_{i(\text { mean })}}\left(\frac{\gamma_{i(a b s)}^{2}}{2}\left(V_{i(r) T} t_{i(0)}\right)^{2}+\frac{\gamma_{i(a b s)}^{2}}{2}\left(c_{i} t_{i(0)}\right)^{2}\right)
$$

Where $M_{i}$ is the particle's mass, $t_{i(\text { mean })=} \frac{h_{i(\text { meam })}}{M_{i(\text { mean })} c_{i(0)(\text { mean })}^{2}}, t_{i(0)}$ is the particle's decay time at rest or internal time unit for stable particles, $V_{i(r) T}$ is the particle's velocity with respect to the expanding universe(or with respect to the end of the universe frame), and

$$
\begin{gathered}
c_{i \mid(a b s)}=\frac{c+\frac{a 1}{f_{r(i)}}}{b 1\left(M_{i(\text { mean })}, V_{i(r) T,}, A_{i(0)}\right)} \\
c_{i(10)}^{i}=\frac{c+\frac{a 1}{f_{r(i)}}}{b 1\left(M_{i(\text { mean })}, A_{i(0)}\right)}
\end{gathered}
$$

And for anti-particles:

$$
\begin{gathered}
c_{i 2(a b s)}=\frac{c+a 2 f_{r(i)}}{b 1\left(M_{i(\text { mean })}, V_{i(r) T,}, A_{i 0)}\right)} \\
c_{i 2(0)}^{i}=\frac{c+a 2 f_{r(i)}}{b 1\left(M_{i(\text { mean })}, A_{i(0)}\right)}
\end{gathered}
$$

and, $f_{r(i)}$ as defined in Brodet (2017b):

$$
f_{r(i)}=g\left(M_{i}\right) e^{-\frac{t_{i}}{\tau}}
$$

and

$$
f_{r(i)(0)}=g\left(M_{i}\right) e^{-\frac{t_{i(0)}}{\tau}}=g\left(M_{i}\right) A_{i(0)}
$$

where $g\left(M_{i}\right)=k_{3}\left(M_{i}+k_{2}\left(M_{i}-\frac{1}{\tau}\right)\right), k_{2}, k_{3}$, a1 and a2 are constants, b1 a function with yet unknown values and

$$
\gamma_{i(a b s)}=\frac{1}{\sqrt{\alpha_{i(a b s)}^{2}-\beta_{i(a b s)}^{2}}}
$$

Where

$$
\alpha_{i(a b s)}=\frac{c_{i 1,2(a b s)}^{2}}{c_{i(0) 1,2}^{2}}, \beta_{i(a b s)}=\frac{V_{i(r) T}^{2}}{c_{i(0) 1,2}^{2}}
$$

In order to better fit current experimental results and better describe the link of a given particle i to its mass group, we may introduce here some changes in the values given above for $c_{i(a b s) 1,2}, c_{i(0) 1,2(a b s)}^{\prime}$ and in $t_{i(\text { mean })}$ and get modified expressions for $P_{i\left(r e s t \_a b s\right)}, P_{i(a b s)}$ such: 


$$
P_{i\left(\text { rest } \_a b s\right)}=\frac{M_{i}}{\tau_{i(\text { mean })}} c_{i(0)}^{2} t_{i(0)}^{2}=\frac{M_{i}}{\tau_{i(\text { mean })}}\left(\frac{\gamma_{i(a b s)}^{2}}{2}\left(V_{i(r) T} t_{i(0)}\right)^{2}-\frac{\gamma_{i(a b s)}^{2}}{2}\left(c_{i} t_{i(0)}\right)^{2}\right)
$$

and

$$
P_{i(a b s)}=\frac{M_{i}}{\tau_{i(\text { mean })}} \gamma_{i(a b s)}^{2} V_{i\left(T\left(V_{r}\right)\right)}^{2} t_{i(0)}^{2}=\frac{M_{i}}{\tau_{i(\text { mean })}}\left(\frac{\gamma_{i(a b s)}^{2}}{2}\left(V_{i(r) T} t_{i(0)}\right)^{2}+\frac{\gamma_{i(a b s)}^{2}}{2}\left(c_{i} t_{i(0)}\right)^{2}\right)
$$

Where $\tau_{i(\text { mean })}$ is the mean lifetime or internal time unit of particle $\mathrm{i}$ and the values of $c_{i(a b s) 1,2}, c_{i(0) 1,2(a b s)}^{\prime}$ are given by:

$$
\begin{gathered}
c_{i 1(a b s)}=\frac{c+\frac{a 1}{f_{r(i)}}}{b 1\left(M_{i}, \tau_{i(\text { mean })}, V_{i(r) T}, A_{i(0)}\right)} \\
c_{i 1(0)}^{i}=\frac{c+\frac{a 1}{f_{r(i)}}}{b 1\left(M_{i}, \tau_{i(\text { mean })}, A_{i(0)}\right)}
\end{gathered}
$$

and for anti-particles:

$$
\begin{gathered}
c_{i 2(a b s)}=\frac{c+a 2 f_{r(i)}}{b 1\left(M_{i}, \tau_{i(\text { mean })}, V_{i(r) T}, A_{i(0)}\right)} \\
c_{i 2(0)}^{i}=\frac{c+a 2 f_{r(i)}}{b 1\left(M_{i}, \tau_{i(\text { mean })}, A_{i(0)}\right)}
\end{gathered}
$$

Consequentially, the values of $\gamma_{i(a b s)}, \alpha_{i(a b s)}, \beta_{i(a b s)}$ changes accordingly in equations 4a,b.

Based on the experimental symmetry between particle/anti-particle momentum and spin we may deduce a symmetry between particle/anti-particle energy and consequently a symmetry in $P_{i(a b s),} P_{i\left(r e s t \_a b s\right)}$ which have the units of angular momentum. Therefore, we may write for specific particle/anti-particle i1 and i2:

$$
\left|P_{i 1 \text { (rest_abs })}\right|=\left|P_{i 2(\text { rest_abs })}\right|=\left|\frac{M_{i 1}}{\tau_{i(\text { mean })}} c_{i 1(0)}^{2} t_{i(0)}^{2}\right|=\left|\frac{M_{i 2}}{\tau_{i(\text { mean })}} c_{i 2(0)}^{2} t_{i(0)}^{2}\right|
$$

Where $P_{i 1(\text { rest_abs })}=-P_{i 2(\text { rest_abs })}$ and the particle $M_{i 1}$ and its anti-particle $M_{i 2}$ are defined using the BreitWigner distribution as discussed in Brodet (2016b) and shown in Figure 1. 


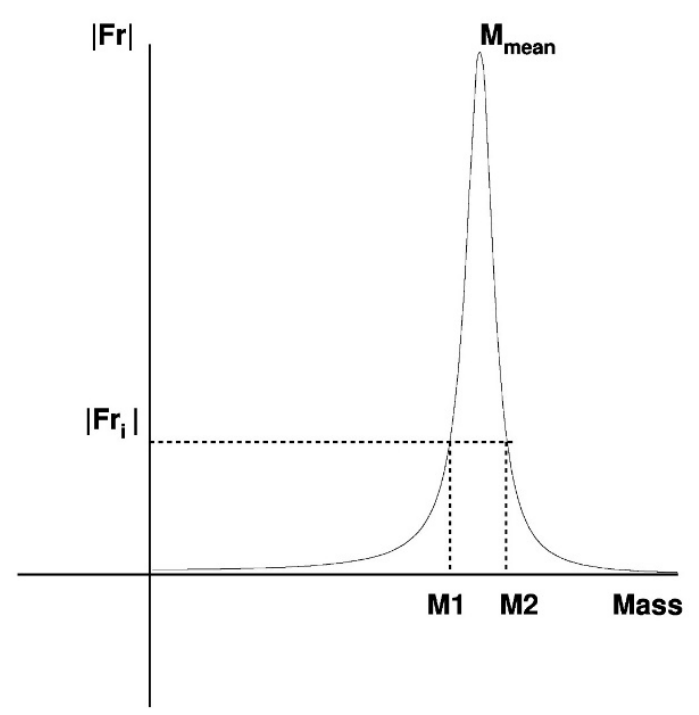

Figure 1. The possible distribution of the particles absolute value of its hidden variable $f_{r}$, as a function of its corresponding Breit-Wigner mass distribution. This is where M1 and M2 describe a particle with mass M1 and its anti-particle with mass M2 which have the same absolute value for their hidden variable, $f_{r(i)}$ and the same absolute energy

Similarly, for $P_{i(a b s)}$ :

$$
\left|P_{i 1(a b s)}\right|=\left|P_{i 2(a b s)}\right|=\left|\frac{M_{i 1}}{\tau_{i(\text { mean })}} \gamma_{i 1(a b s)}^{2} V_{i 1(T)}^{2} t_{i(0)}^{2}\right|=\left|\frac{M_{i 2}}{\tau_{i(\text { mean })}} \gamma_{i 2(a b s)}^{2} V_{i 2(T)}^{2} t_{i(0)}^{2}\right|
$$

Where $P_{i 1(a b s)}=-P_{i 2(a b s)}$.

Therefore, as shown in Brodet (2018), we may take the total derivative based on equation $4 \mathrm{~b}$ and identify the absolute energy and momentum:

$$
\begin{gathered}
E_{i(a b s)}=\gamma_{i(a b s)}^{2} M_{i} c_{i}^{2} \frac{t_{i(0)}}{\tau} \\
p p_{i(a b s)}=\gamma_{i(a b s)}^{2} M_{i} V_{i(r) T} \frac{t_{i(0)}}{\tau}
\end{gathered}
$$

Note that both $P_{i(a b s),} P_{i\left(r e s t \_a b s\right)}$ are defined as positions in the angular dimension which is regarded here to be the most suitable for such absolute position definitions as it may include the relevant variables in a fundamental way. 2.2 Deconstruction of $P_{i(a b s)}$, Parity and CP Violation

The expressions for $P_{i(a b s)}$ in equations 4a,b uses four independent variables to minimally describe the particle's absolute position and absolute position at rest. These four variables are the hidden variable in time $f_{r(i)}$, the particle's velocity with respect to the expanding universe $V_{i(r) T}$, the particle's mean lifetime $\tau_{i(\text { mean })}$ that 
indicate which mass group the particle's belongs to and $A_{i(0)}$, the particle's exponential phase at $t_{i(0)}$ defined in equation $2 \mathrm{f}$.

As discussed in Brodet (2017a), the particle's absolute position may originate from a complex number in space and time and then may be extended to a complex number in angular momentum. Therefore, the expression for $P_{i(a b s)}$ may be expressed as the absolute value of the complex number:

$$
\left|z^{2}\right|=P_{i(a b s)}=\frac{M_{i}}{2 \tau} \gamma_{i(a b s)}^{2} V_{i(T)}^{2} t_{i(0)}^{2}=\frac{M_{i} \gamma_{i(a b s)}^{2}}{2 \tau}\left(V_{r(i) T} t_{i(0)}\right)^{2}+\frac{M_{i} \gamma_{i(a b s)}^{2}}{2 \tau}\left(C_{i} t_{i(0)}\right)^{2}
$$

$V_{(i) T}=\sqrt{V_{r(i) T}^{2}+C_{i}^{2}}$ where $V_{(i) T}$ is the total velocity given by:

Therefore, we may get:

$$
z=\sqrt{\frac{M_{i}}{2 \tau}}\left(\gamma_{i(a b s)} V_{r(i) T} t_{i(0)}\right)+i \sqrt{\frac{M_{i}}{2 \tau}}\left(\gamma_{i(a b s)} C_{i} t_{i(0)}\right)
$$

Which is equal to:

$$
Z_{i}=(a c e-b d e-a d f-b c f)+i(a c f-b d f+a d e+b c e)
$$

Where

$$
\begin{aligned}
z 1 & =a-i b \\
z 2 & =c+i d \\
z 3 & =e+i f
\end{aligned}
$$

Therefore, we may deconstruct into three complex number such:

$$
\begin{gathered}
z 1=\gamma_{i(a b s)} t_{i(0)} / \sqrt{2}-i \gamma_{i(a b s)} t_{i(0)} / \sqrt{2} \\
z 2=\frac{1}{\sqrt{2}} \sqrt{\frac{M_{i}}{2 \tau}}+i \frac{1}{\sqrt{2}} \sqrt{\frac{M_{i}}{2 \tau}} \\
z 3=V_{r(i) T)}+i C_{i}
\end{gathered}
$$

Which gives:

$$
P_{i(a b s)}=\left|z^{2}\right|=\left|z 1^{2}\right| \cdot\left|z 2^{2}\right| \cdot\left|z 3^{2}\right|=\frac{M_{i}}{2 \tau} \gamma_{i(a b s)}^{2} V_{(i) T}^{2} t_{i(0)}^{2}
$$

Therefore, we can see that at the end of the universe frame (i.e. a frame which placed at the edge of the expanding universe where $\gamma_{i(a b s)}=1$ ), the complex number of time, z1, is symmetric between particle i1 and anti-particle i2 in the operation $f_{r}$ to $-f_{r}$

$$
\left|z 1_{i 1}^{2}\right|=\left|z 1_{i 2}^{2}\right|
$$

and 


$$
\left|t_{i 1(0)}^{2}\right|=\left|t_{i 2(0)}^{2}\right|=\left|t_{i(0)}^{2}\right|
$$

Also in the above frame, the mean lifetime and the velocity complex numbers are asymmetric where

$$
\begin{aligned}
& \left|z 2_{i 1}^{2}\right| \neq\left|z 2_{i 2}^{2}\right| \\
& \left|\frac{M_{i 1}}{2 \tau}\right| \neq\left|\frac{M_{i 2}}{2 \tau}\right|
\end{aligned}
$$

and

$$
\begin{aligned}
& \left|z 3_{i 1}^{2}\right| \neq\left|z 3_{i 2}^{2}\right| \\
& \left|V_{i 1(T)}^{2}\right| \neq\left|V_{i 2(T)}^{2}\right|
\end{aligned}
$$

The above possible asymmetry may have interesting implications on parity violation results as experimentally known for example in the process $e^{+} e^{-} \rightarrow \mu^{+} \mu^{-}$at LEP. Therefore, if we consider the $e^{+} e^{-}$initial conditions at the LEP experiment, the $e^{+} e^{-}$are assumed to have the same initial energy as they are accelerated to the same velocity before they interact. However, if we assume the energy and momentum expressions given in equations $7 \mathrm{a}, 7 \mathrm{~b}$ are correct, then in order for the initial $e^{+} e^{-}$pair to have the same momentum:

$$
\left|p p_{i\left(e^{+}\right)(a b s)}\right|=\left|p p_{i\left(e^{-}\right)(a b s)}\right|
$$

It means the $e^{+}$and $e^{-}$will have different velocities as they may have slightly different masses such:

$$
\gamma_{i\left(e^{+}\right)(a b s)}^{2} M_{i\left(e^{+}\right)} V_{i\left(e^{+}\right)(r) T} \frac{t_{i(0)}}{\tau}=\gamma_{i\left(e^{-}\right)(a b s)}^{2} M_{i\left(e^{-}\right)} V_{i\left(e^{-}\right)(r) T} \frac{t_{i(0)}}{\tau}
$$

In practice, the $e^{+} e^{-}$resonance is very narrow, which means that the possible mass difference between the $e^{+}$ and $e^{-}$can be very small and therefore their velocity difference may be very small as well. However, even a very small velocity difference may have a non-negligible effect on the $\gamma_{i\left(e^{+}\right)(a b s)}$ and $\gamma_{i\left(e^{-}\right)(a b s)}$ factors of the $e^{+}$and $e^{-}$and therefore on the $e^{+}$and $e^{-}$initial momentum. Therefore, in the case of the LEP experiment, where the $e^{-}$and $e^{+}$velocities are the same, there may be a small energy and momentum difference between the incoming $e^{-}$and $e^{+}$pair. Moreover, the asymmetric incoming $e^{-}$and $e^{+}$momentum may define asymmetric initial angular momentum $l_{i\left(e^{+}\right)}=p p_{i\left(e^{+}\right)(a b s)} X_{i\left(e^{+}\right)}$and $l_{i\left(e^{-}\right)}=p p_{i\left(e^{-}\right)(a b s)} X_{i\left(e^{-}\right)}$where $X_{i\left(e^{+}\right)}=X_{i\left(e^{-}\right)}$and $X_{i\left(e^{+}\right)}$and $X_{i\left(e^{-}\right)}$describes the position of the $e^{+}$and $e^{-}$in the Gaussian shape of the incoming beams of $e^{+}$and $e^{-}$as was discussed in Brodet (2016a) and schematically shown in Figure 2. Therefore, as the total angular momentum should be conserved, $l_{i\left(e^{+} e^{-}\right)}=l_{i\left(e^{+}\right)}+l_{i\left(e^{-}\right)}$, the above small 
asymmetry difference between $e^{+}$and $e^{-}$should give some small, non-zero $l_{i\left(e^{+} e^{-}\right)}$that should be translated to the outgoing $\mu^{+} \mu^{-}$. Thus, the momentum of the $\mu^{+} \mu^{-}$may be equal and opposite but there may be an asymmetry in their sum of angular momentum, $l_{i\left(\mu^{+} \mu^{-}\right)}$, which would be expressed in their position in the outgoing hypothetical Gaussian beams, $X_{i\left(\mu^{+}\right)}$and $X_{i\left(\mu^{-}\right)}$. These possible differences between $X_{i\left(\mu^{+}\right)}$and $X_{i\left(\mu^{-}\right)}$may be expressed in a forward-backward asymmetry in the $\mu^{+} \mu^{-}$angular production as observed in LEP. It is suggested that in case the momentum difference between the initial $e^{+} e^{-}$is large, the outgoing muons may be then boosted in the direction of the initial $e^{+} e^{-}$momentum difference because the muons $X_{i\left(\mu^{+}\right)}$and $X_{i\left(\mu^{-}\right)}$values may not be able to fully absorb the initial $e^{+} e^{-}$large angular momentum value $l_{i\left(e^{+} e^{-}\right)}$.

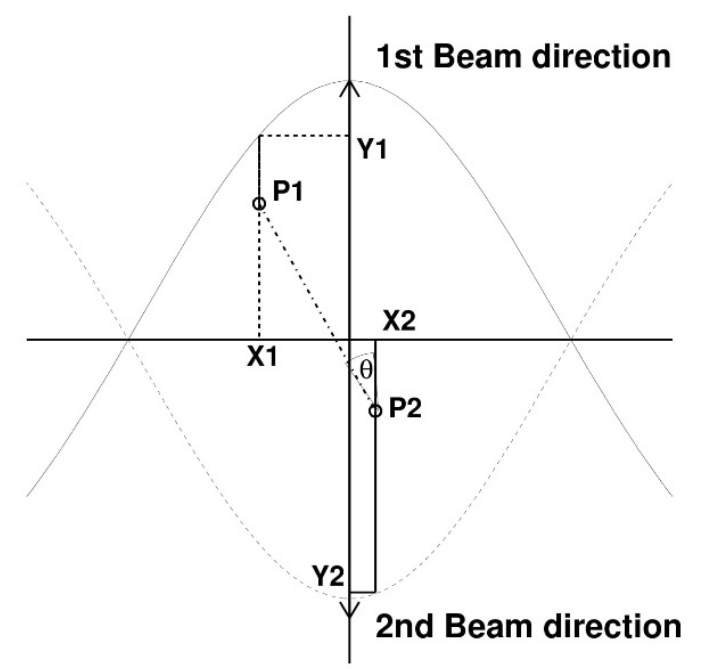

Figure 2. Possible 2-Dimentional description of $e^{+} e^{-}$interaction, where the beam of $e^{+}$are described by beam 1 and the beam of $e^{-}$are described by beam 2. This is where particle 1 in beam 1 has a location X1 and particle 2 in beam 2 has location X2 in the beams Gaussian shape. P1 and p2 describes the momentum. Note that in section 2.2 it was suggested that $\mathrm{X} 1=\mathrm{X} 2$ for the initial $e^{+} e^{-}$in an annihilation process

The possible particle/anti-particle asymmetry discussed above, may be used to explain not only parity violation processes but may also be a part of a possible explanation for the $\mathrm{CP}$ violation observation such as seen in the neutral Kaon system. Therefore, instead of suggesting a $K 0-\bar{K} 0$ superposition we may alternitevly suggest that the $K 0$ and the $\bar{K} 0$ are in fact different mesons with different rest mass and different mean lifetime's. This is where the Breit-Wigner of the s and d quarks may be interpreted to give an asymmetric value for the $s$ and $\bar{s}$ masses and similarly for the $d$ and $\bar{d}$ masses as first suggested in Brodet (2017b). Therefore, these different 
quark masses would give a different rest mass for the $K 0$ and $\bar{K} 0$. In this context, if we let $K 0_{s}=\mathrm{K}$ short and $\bar{K} 0_{l}=\mathrm{K}$ long, we get $M_{i\left(k 0_{s}\right)} \neq M_{i\left(\bar{k} 0_{l}\right)}$ which may yield the two different mean lifetimes, $\tau_{\text {short }}$ and $\tau_{\text {long }}$. Therefore, the absolute energy at rest

of $K 0_{s}$ and $\bar{K} 0_{l}$ is not equal such:

$$
E_{i\left(k 0_{s}\right)} \neq E_{i\left(\bar{k} 0_{l}\right)}
$$

Where,

$$
\begin{gathered}
E_{i\left(k 0_{s}\right)}=\gamma_{i\left(k 0_{s}\right)(a b s)}^{2} M_{i\left(k 0_{s}\right)} c_{i(k 0)}^{2} \frac{t_{i\left(h 0_{s}\right)}}{\tau_{\text {short }}} \\
E_{i\left(\bar{k} 0_{l}\right)}=\gamma_{i\left(\bar{k} 0_{l}\right)(a b s)}^{2} M_{i\left(\bar{k} 0_{l}\right)} c_{i\left(k 0_{l}\right)}^{2} \frac{t_{i\left(h 0_{l}\right)}}{\tau_{\text {long }}}
\end{gathered}
$$

and if $K 0=\mathrm{K}$ long and $\bar{K} 0=\mathrm{K}$ short, then the $E_{i\left(k 0_{s}\right)}$ and $E_{i\left(\bar{k} 0_{l}\right)}$ in equations $13 \mathrm{~b}, 13 \mathrm{c}$ should be replaced. The assignment of $K 0, \bar{K} 0, \mathrm{~K}$ short and $\mathrm{K}$ long may have to be done according to the experimental data. Therefore, the different decay times of $K 0_{s}$ and $\bar{K} 0_{l}$ may affect their different absolute rest energies of $E_{i\left(k 0_{s}\right)}$ and $E_{i\left(\bar{k} 0_{l}\right)}$. Thus, this may mean that the length of time necessary to measure these particles energy and momentum may also be different. This aspect will be discussed in section 3. Also, as the energies $E_{i\left(k 0_{s}\right)}$ and $E_{i\left(\bar{k} 0_{l}\right)}$ may not be equal, the process of $K 0-\bar{K} 0$ oscillation may not occur between $E_{i\left(k 0_{s}\right)}$ and $E_{i\left(\bar{k} 0_{l}\right)}$. It is possible however, that instead an oscillation may occur between $k 0_{s}$ and $\bar{k} 0_{s}$ which may have different rest masses but the same mean lifetime and the same absolute rest energy. Similarly, an oscillation may occur between $\bar{k} 0_{l}$ and $k 0_{l}$. Note that the mass of $\bar{k} 0_{s}$ may be different then the mass of $\bar{k} 0_{l}$ and similarly the mass of $k 0_{l}$ may be different then the mass of $k 0_{s}$. i.e. there may be four different mean rest masses associated with the neutral kaon system. The above four different masses may emerge from the possible different $\mathrm{d}$ and $\bar{d}$ (and s and $\bar{S}$ ) masses discussed previously and from possible different $f_{r(i)}$ distribution of the $\mathrm{s}$ and $\mathrm{d}$ quarks containing the $k 0_{s}, \bar{k} 0_{s}$ particles and the $k 0_{l}, \bar{k} 0_{l}$ particles. Possible experimental evidence for the above will be discussed in section 3 . 


\subsection{The Particle Existence Time}

Let us consider the time complex number, $z 1$, defined in section 2.2. The absolute value of $z 1$ is given by:

$$
z 1=\sqrt{\left(\frac{\gamma_{i(a b s)} t_{i(0)}}{\sqrt{2}}\right)^{2}+\left(\frac{\gamma_{i(a b s)} t_{i(0)}}{\sqrt{2}}\right)^{2}}=\gamma_{i(a b s)} t_{i(0)}
$$

As $\left|z 1^{2}\right|$ is a part of $P_{i(a b s)}$, we may identify it as the absolute particle's decay time or absolute particle internal time unit and draw from it the particle's internal frequency, $\frac{1}{z 1}$, which we may identify as a quantity that is equal to the previously define hidden variable in time $f_{r}$. Therefore:

$$
f_{r(i)}=\frac{1}{\gamma_{i(a b s)} t_{i(0)}}
$$

The expression for $f_{r}$ given in equation $14 \mathrm{~b}$ above, should be equal to the previous expression for $f_{r}$ given in equation $2 \mathrm{f}$ and in Brodet (2010), LEP of:

$$
f_{r(i)}=g\left(M_{i}\right) e^{-\frac{t_{i}}{\tau}}
$$

Where from Brodet (2010),

$$
t_{i}=t_{i(0)}\left(1+\beta_{i}^{/ 2}\right)
$$

Where $\beta_{i}^{\prime}=\frac{V_{i(r) T}}{C_{i}}$

Therefore:

$$
\left|\frac{1}{\gamma_{i(a b s)} t_{i(0)}}\right|=\left|f_{i(r)}\right|=\left|g\left(M_{i}\right) e^{-\frac{t_{i(0)}\left(1+\beta_{i}^{2}\right)}{\tau}}\right|
$$

Therefore:

$$
\gamma_{i(a b s)} f_{r(i)} t_{i(0)}=1
$$

Which describes the sum of the particle's internal changes or the particle's existence time as defined in Brodet (2018). Therefore, from equation 16, we may draw the interesting conclusion that the particles existence time is constant for all the fundamental particles. Also we see that:

$$
\gamma_{i(a b s)}=1+\beta_{i}^{\prime 2}
$$

\section{Possible Experimental Investigation}

If the above is correct, there may be other experimental manifestations for it. Therefore, let us consider again the process $e^{+} e^{-} \rightarrow \mu^{+} \mu^{-}$in the LEP experiment. As was discussed in section 2.2, there may be an angular momentum asymmetry in $l_{i\left(\mu^{+} \mu^{-}\right)}$but the outgoing $\mu^{+} \mu^{-}$may have the same momentum, $\left|p p_{i\left(\mu^{+}\right)}\right|=\left|p p_{i\left(\mu^{-}\right)}\right|$. 
Therefore, the outgoing velocities of the muon anti-muon pair, $V_{i\left(\mu^{+}\right)(r) T}$ and $V_{i\left(\mu^{-}\right)(r) T}$ may be different and satisfy:

$$
\gamma_{i\left(\mu^{+}\right)(a b s)}^{2} M_{i\left(\mu^{+}\right)} V_{i\left(\mu^{+}\right)(r) T} \frac{t_{i(0)\left(\mu^{+}\right)}}{\tau}=\gamma_{i\left(\mu^{-}\right)(a b s)}^{2} M_{i\left(\mu^{-}\right)} V_{i\left(\mu^{-}\right)(r) T} \frac{t_{i(0)\left(\mu^{-}\right)}}{\tau}
$$

Equation 18a describes the possible absolute momentum of the $\mu^{+} \mu^{-}$. In an experiment like Delphi Collaboration (1988) at LEP, the $\mu^{+} \mu^{-}$are measured with respect to the lab center of mass frame. Therefore, the $\mu^{+} \mu^{-}$absolute momentum should be transformed into the lab center of mass frame which changes equation 18a into:

$$
\gamma_{i\left(\mu^{+}\right)} \gamma_{i\left(\mu^{+}\right)(a b s)}^{2} M_{i\left(\mu^{+}\right)} V_{i\left(\mu^{+}\right)(r) T} \frac{t_{i(0)\left(\mu^{+}\right)}}{\tau_{\mu}}=\gamma_{i\left(\mu^{-}\right)} \gamma_{i\left(\mu^{-}\right)(a b s)}^{2} M_{i\left(\mu^{-}\right)} V_{i\left(\mu^{-}\right)(r) T} \frac{t_{i(0)\left(\mu^{-}\right)}}{\tau_{\mu}}
$$

Where

$$
\begin{gathered}
\gamma_{i\left(\mu^{+}, \mu^{-}\right)}=\frac{1}{\sqrt{\alpha_{i\left(\mu^{+}, \mu^{-}\right)}^{2}-\beta_{i\left(\mu^{+}, \mu^{-}\right)}^{2}}} \\
\alpha_{i\left(\mu^{+}, \mu^{-}\right)}=\frac{c_{i\left(\mu^{+}, \mu^{-}\right)}^{2}}{c_{i(0)\left(\mu^{+}, \mu^{-}\right)}^{2}}, \beta_{i\left(\mu^{+}, \mu^{-}\right)}=\frac{V_{i(r)\left(\mu^{+}, \mu^{-}\right)}^{2}}{c_{i(0)\left(\mu^{+}, \mu^{-}\right)}^{2}}
\end{gathered}
$$

Where $V_{i(r)\left(\mu^{+}\right)}$and $V_{i(r)\left(\mu^{-}\right)}$are the $\mu^{+} \mu^{-}$velocities with respect to the lab center of mass frame and $V_{i\left(\mu^{+}\right)(r) T}$ and $V_{i\left(\mu^{+}\right)(r) T}$ are the $\mu^{+} \mu^{-}$velocities with respect to the end of the universe frame as defined in section 2.1 and $C_{i}^{\prime \prime}$ is a different function then $C_{i}$.

The velocities of the $\mu^{+}$and $\mu^{-}$may not only be different but may also correlated. Therefore, it may be possible to attempt to search for a velocity difference and a correlation between $V_{i(r)\left(\mu^{+}\right)}$and $V_{i(r)\left(\mu^{-}\right)}$in $e^{+} e^{-} \rightarrow \mu^{+} \mu^{-}$events and test the above suggestion. This may be done, using the data of the DELPHI experiment at LEP where there was a Cherenkov detector that measured particles velocity.

Another option to test the above expression for the particle's momentum, would be to investigate the process $e^{+} e^{-} \rightarrow \tau^{+} \tau^{-}$at LEP. In this process, one may attempt to search for a possible correlation between $\tau^{+}$and $\tau^{-}$in $\tau^{+} \tau^{-}$events. This is where in each $\tau^{+} \tau^{-}$event, the momentum of the $\tau^{+}$and $\tau^{-}$should have an equal absolute value such:

$$
\gamma_{i\left(\tau^{+}\right)(a b s)}^{2} M_{i\left(\tau^{+}\right)} V_{i\left(\tau^{+}\right)(r) T} \frac{t_{i(0)\left(\tau^{+}\right)}}{\tau}=\gamma_{i\left(\tau^{-}\right)(a b s)}^{2} M_{i\left(\tau^{-}\right)} V_{i\left(\tau^{-}\right)(r) T} \frac{t_{i(0)\left(\tau^{-}\right)}}{\tau}
$$

Similarly to the $\mu^{+} \mu^{-}$muons case discussed earlier, in the lab frame the $\tau^{+} \tau^{-}$momentum may be measured: 


$$
\gamma_{i\left(\tau^{+}\right)} \gamma_{i\left(\tau^{+}\right)(a b s)}^{2} M_{i\left(\tau^{+}\right)} V_{i\left(\tau^{+}\right)(r) T} \frac{t_{i(0)\left(\tau^{+}\right)}}{\tau}=\gamma_{i\left(\tau^{-}\right)} \gamma_{i\left(\tau^{-}\right)(a b s)}^{2} M_{i\left(\tau^{-}\right)} V_{i\left(\tau^{-}\right)(r) T} \frac{t_{i\left(0\left(\tau^{-}\right)\right.}}{\tau}
$$

Therefore, there may be correlation between the decay times, $t_{i\left(\tau^{+}\right)}$and $t_{i\left(\tau^{-}\right)}$of the $\tau^{+}$and $\tau^{-}$in each event.

This is where $t_{i\left(\tau^{+}\right)}=\gamma_{i\left(\tau^{+}\right)(a b s)} t_{i\left(\tau^{+}\right)(0)}$ and $t_{i\left(\tau^{-}\right)}=\gamma_{i\left(\tau^{-}\right)(a b s)} t_{i\left(\tau^{-}\right)(0)}$. Note that $t_{i\left(\tau^{+}\right)}$may not necessarily equal to $t_{i\left(\tau^{-}\right)}$as although $t_{i\left(\tau^{+}\right)(0)}$ should be equal to $t_{i\left(\tau^{+}\right)(0)}$, their boost factors, $\gamma_{i\left(\tau^{+}\right)(a b s)}$ and $\gamma_{i\left(\tau^{-}\right)(a b s)}$ may not be equal to each other.

Such a correlation may be searched for using DELPHI or any other LEP experiment data sets, under the limitation of the uncertainty principle as discussed in Brodet (2016a, 2016b). This is where, according to Brodet (2016a, 2016b) the longer decay times may be measured better than the short ones.

A correlation found in the searches above would support the momentum expression given in equation $7 \mathrm{~b}$ and its associated particle/anti-particle symmetry and asymmetry and may suggest a deterministic link between the particle/anti-particle exponential distribution and the corresponding Breit-Wigner distribution. Such a deterministic link may support the idea of a hidden variable in time that connects the distributions and may be responsible for the particle/anti-particle decay.

As was discussed in section 2.2, the absolute rest energies of $\mathrm{k}$ short and $\mathrm{k}$ long may be different. Since this possible difference is suggested to be related to the difference in the $\mathrm{k}$ short $/ \mathrm{k}$ long decay times, it would be possible to attempt to modify detectors such as NA62 to prolong the length of time of the energy and momentum measurements. This is where the layers in the calorimeter may be extended while the blocking material (usually iron) layers may be reduced such that the particles will loss their energy through a much longer length of time. In the momentum measurement, one can extend the magnets size which will allow to increase the reconstruction of the particles curvature and follow the particles over a larger length of time. Therefore, the kaons rest energy may be reconstructed from the above energy and momentum measurements. It is suggested that such a modified detector, may measure an energy difference at rest between $\mathrm{k}$ short and $\mathrm{k}$ long and support the suggestion described in section 2.2 regarding the neutral kaon system.

\section{Conclusions}

The possible particle/anti-particle hidden symmetry in time and the possible particle/anti-particle asymmetry in mass and velocity were discussed. Consequently, the particle/anti-particle absolute positions were deconstructed into three complex numbers, containing three Independent characteristics of the particle/anti-particle namely, internal time, mean lifetime and velocity. The possible particle/anti-particle hidden symmetry in time was demonstrated in the time complex number. The possible particle/anti-particle asymmetry in mass and velocity was demonstrated in the mean lifetime and velocity complex numbers. The implication of the above asymmetry was discussed in the context of $e^{+} e^{-} \rightarrow Z / \gamma^{*} \rightarrow \mu^{+} \mu^{-}$process and the parity violation observed in this process was attempted to be explained using the suggested asymmetry in mass and velocity. Moreover, it was suggested that the mass asymmetry may be the origin of the $\mathrm{CP}$ violation in the neutral Kaon system. Finally, experimental ways to investigate and test the above were presented.

\section{Conflict of interests}

The authors declare that there is no conflict of interests regarding the publication of this paper.

\section{References}

Brodet, E. (2010). The possibility of a hidden variable in time. Physics Essays, 23(4).

Brodet, E. (2016a). The relationship between possible hidden variables in time and space and the particle's angular momentum and wave function. Physics Essays, 29(3).

Brodet, E. (2016b). The relationship between the possibility of a hidden variable in time and the uncertainty principle. Progress in Physics, 1(3).

Brodet, E. (2017a). The total energy and momentum stored in a particle. Applied Physics Research, 9(2).

Brodet, E. (2017b). Possible hidden symmetry in time.Physics Essays, 30(4). 
Brodet, E. (2018). Absolute position and energy. Applied Physics Research, 10(4).

Delphi Collaboration. (1988). Nuclear instruments and methods in physics research, section A, 270(2-3).

LEP. Large Electron Positron. Retrieved from https://home.cern/science/accelerators/large-electron- positroncollider

NA62 Detector. Retrieved from https://home.cern/science/experiments/na62

\section{Copyrights}

Copyright for this article is retained by the author(s), with first publication rights granted to the journal.

This is an open-access article distributed under the terms and conditions of the Creative Commons Attribution license (http://creativecommons.org/licenses/by/4.0/). 